\title{
CensNet: Convolution with Edge-Node Switching in Graph Neural Networks
}

\author{
Xiaodong Jiang ${ }^{1,2}$, Pengsheng $\mathbf{J i}^{2}$ and Sheng $\mathbf{L i}^{1}$ \\ ${ }^{1}$ Department of Computer Science, University of Georgia, Athens, GA, USA \\ ${ }^{2}$ Department of Statistics, University of Georgia, Athens, GA, USA \\ \{xiaodong, psji, sheng.li\}@uga.edu
}

\begin{abstract}
In this paper, we present CensNet, Convolution with Edge-Node Switching graph neural network, for semi-supervised classification and regression in graph-structured data with both node and edge features. CensNet is a general graph embedding framework, which embeds both nodes and edges to a latent feature space. By using line graph of the original undirected graph, the role of nodes and edges are switched, and two novel graph convolution operations are proposed for feature propagation. Experimental results on real-world academic citation networks and quantum chemistry graphs show that our approach has achieved or matched the state-of-the-art performance.
\end{abstract}

\section{Introduction}

Deep learning models like convolutional neural networks (CNN) have been remarkably successful in many domains [LeCun et al., 2015], including computer vision, natural language processing, signal processing, etc. In particular, $\mathrm{CNN}$ and its variants are capable of extracting multi-scale localized spatial features and producing highly expressive representations [Krizhevsky et al., 2012; He et al., 2016]. Instead of using sophisticated feature engineering procedures that heavily rely on domain expertise, $\mathrm{CNN}$ based models are usually trained in an end-to-end fashion, and they can model the high-order interactions among input features for specific tasks. The convolution operations in $\mathrm{CNN}$ are well defined on data with underlying Euclidean structures (e.g., images), but they cannot be directly generalized to non-Euclidean data such as graphs and manifolds [Bronstein et al., 2017].

Graph-structured data is ubiquitous, from social network platforms to citation and co-authorship relations, from protein-protein interactions to chemical molecules $[\mathrm{Li}$ and Fu, 2015; Ji and Jin, 2016; Li et al., 2017]. Graph, as a complex data structure, is very effective in describing the relationships (edges) of objects (nodes). Due to the expressive power and flexibility of graph-structured data, graph neural networks (GNN) have attracted increasing attention in recent years, which try to adapt the effective deep representation learning approaches from Euclidean to non-Euclidean domains [Zhou et al., 2018]. The earliest GNN method might be traced back to the work in [Scarselli et al., 2009], which extends the general neural networks to graph domain. Along this research direction, many other GNN models have been proposed recently, such as the ChebNet [Defferrard et al., 2016a], graph convolutional networks (GCN) [Kipf and Welling, 2017], GraphSAGE [Hamilton et al., 2017], Lanczosnet [Liao et al., 2019], etc. By leveraging the node adjacency matrix, these GNN models analogously define convolution operators on graphs in either spectral or spatial spaces and have obtained promising performance in tasks like node classification [Zhou et al., 2018]. With a few notable exceptions [Monti et al., 2017; Veličković et al., 2018; Schlichtkrull et al., 2018], GNN methods mainly focus on obtaining effective node embeddings, but ignore the information associated with edges that can be beneficial to many tasks such as node or edge classification, link prediction, community detection, and regression.

In this paper, we aim to learn both node embeddings and edge embeddings for graphs. Graphs in reality usually contain both node and edge features. In social networks, the node features could be demographic information or user behaviors, while the edge features might be the type of relationships or the years of friendship. In citation networks, the node features could be document-level embeddings of papers, and the edge features might indicate the common keywords or co-authors of two articles. In chemistry domain, each compound can be considered as a graph, where atoms are nodes, and properties of chemical bonds are edge features. We justify the motivation of jointly learning node and edge embeddings from the following two aspects. First, it is clear that edge and nodes always provide complementary feature information, which will be helpful for graph embedding. Second, learning edge embeddings is essential for edge-relevant tasks, such as edge classification and regression.

Inspired by the Line Graph in graph theory [Harary and Norman, 1960], we propose a novel convolution with edgenode switching network (CensNet) for learning node and edge embeddings. Let $G$ denote the node adjacency matrix of a graph, its line graph $L(G)$ can be constructed to represent the adjacencies between edges of $G$. In our framework, the role of node and edge can be switched, and CensNet conducts the graph convolution operations on both the input graph $G$ and its line graph counterpart. With the help of node and edge features, CensNet employs two forward-pass feature propa- 
gation rules on $G$ and $L(G)$ to alternatively update the node and edge embeddings. Also, a mini-batch training algorithm for CensNet is devised to handle large-scale graphs.

The main contributions of this work are summarized below.

- Co-embedding of nodes and edges. The proposed CensNet is a general graph embedding framework which can embed both nodes and edges to a latent feature space simultaneously, with the help of graph structure and node/edge features. Moreover, the node and edge embeddings can be mutually enriched owing to the alternative update rules.

- Convolution on line graph. We design convolution operations on both the input graph and its line graph counterpart, where the role of node and edge are switched.

- Diverse learning tasks on graphs. We apply CensNet to several graph-based learning tasks, including multitask graph classification, graph regression, and semisupervised node classification.

- Extensive evaluations on benchmark data sets. The extensive experiments on citation networks (Cora, Citeseer [Sen et al., 2008], and PubMed [Namata et al., 2012]) and Quantum Chemistry data sets (Tox21 and Lipophilicity [Wu et al., 2018]) show that our method has achieved or matched state-of-the-art performance.

\section{Preliminaries}

\subsection{Notations}

We present the mathematical notations for a graph with node and edge features in the following list.

- We assume an undirected graph $G$ with node set $V$ and edge set $E, E \subseteq V \times V . N_{v}=|V|$ and $N_{e}=|E|$ denote the number of nodes and edges, respectively.

- Let $A_{v} \in \mathbb{R}^{N_{v} \times N_{v}}$ be the adjacency matrix of $G$, where each element $A_{v}(i, j)$ denotes the connectivity of node $i$ and node $j$, where $i, j \in\left\{1,2, \ldots, N_{v}\right\} . A_{v}$ is a binary matrix in unweighted graph.

- Let $X \in \mathbb{R}^{N_{v} \times d_{v}}$ be the node feature matrix, where each node is associated with a $d_{v}$-dimensional feature vector.

- Let $A_{e} \in \mathbb{R}^{N_{e} \times N_{e}}$ be the binary edge adjacency matrix of $G$, or node adjacency matrix of $L(G) . A_{e}(m, n)$ equals to 1 if the edge $m$ and edge $n$ are connected by a node in $G$, otherwise 0 , where $m, n \in\left\{1,2, \ldots, N_{e}\right\}$.

- Let $Z \in \mathbb{R}^{N_{e} \times d_{e}}$ denote the edge feature matrix, where each edge has a $d_{e}$-dimensional feature vector.

- Let $H_{v}^{(l)}$ be the $l$-th hidden layer of node convolution with $H_{v}^{(0)}=X$; Define $H_{e}^{(l)}$ as the $l$-th layer of edge convolution, and $H_{e}^{(0)}=Z$.

We will also introduce other notations in the rest of the paper when necessary.

\subsection{Graph Convolution and Embedding}

Given a graph $G$ and its corresponding node feature matrix $X$, there are two major graph convolutions in literature, spectral convolution in the Fourier domain and spatial convolution in the node (or vertex) domain. We discard the subscripts of our notations for a moment, assuming $X$ is the node feature matrix, and $A$ is the node adjacency matrix.

Spectral graph convolution. A spectral graph convolution is defined as the multiplication of a signal with a filter in the Fourier domain of the graph. The graph Fourier transform $Y$ is defined as the multiplication of a graph signal (e.g., node features $X$ ) with the eigenvector matrix $U$ of the graph Laplacian $L$, i.e., $Y=U^{T} X$ and $U \Sigma U^{T}=L$. The graph Laplacian $L$ can be defined in different ways: the simple Laplacian $D-A$, the symmetric normalized Laplacian $I-D^{-\frac{1}{2}} A D^{-\frac{1}{2}}$, or the random walk Laplacian $I-D^{-1} A$, where $I$ is an identity matrix and $D$ is the diagonal degree matrix. The symmetric normalized Laplacian is usually desirable due to the nice properties including symmetric, positive semidefinite, and all eigenvalues are in $[0,2]$. The ChebyNet [Defferrard et al., 2016a] and GCN [Kipf and Welling, 2017] are based on spectral graph convolutions. The GCN proposes a layerwise propagation rule based on an approximated graph spectral kernel as follows

$$
H^{(l+1)}=\sigma\left(\tilde{D}^{-\frac{1}{2}} \tilde{A} \tilde{D}^{-\frac{1}{2}} H^{(l)} W^{(l)}\right)
$$

where $\tilde{A}=A+I$ is the adjacency matrix with selfconnections, $\tilde{D}$ is the degree matrix, $H^{(l)}$ and $W^{(l)}$ are the hidden feature matrix and learnable weight in the $l$-th layer.

Spatial graph convolution. A spatial graph convolution is defined on the node domain, which can integrate or aggregate the signals among its neighbor nodes. MoNet [Wu et al., 2018] and GraphSAGE [Hamilton et al., 2017] are aggregation based representation learning models in this direction.

Node and Edge Embeddings. There have only been a few scattered examples that can link both node and edge features in a graph convolution simultaneously, which can be categorized as two approaches. The first one is to define different weight matrices for each relation or dimension on the edge features and aggregate different relation propagation in an additive fashion. [Schlichtkrull et al., 2018] used the following rule for the forward-pass update

$$
h_{i}^{l+1}=\sigma\left(\sum_{r \in \mathcal{R}} \sum_{j \in \mathcal{N}_{i}^{r}} \frac{1}{c_{i, r}} W_{r}^{(l)} h_{j}^{(l)}+W_{0}^{(l)} h_{i}^{(l)}\right)
$$

where each relation $r \in \mathcal{R}$ has its corresponding weight matrix $W_{r}$. Such a simple aggregation enjoys the efficiency in computation but cannot capture the dependence of different relations and the interaction between the node and edge features. The second approach is to add the attention mechanism to graph convolutions by specifying different weights to different nodes in a neighborhood. MonNet [Monti et al., 2017] and GAT [Veličković et al., 2018] are examples along this line. More importantly, both of these two approaches can only handle discrete or low-dimensional edge features.

Different from existing graph convolution and embedding methods, the proposed CensNet framework is independent of the choice of graph convolution, although we only implement one representative spectral-based method [Kipf and Welling, 2017] in this paper. Moreover, the co-embedding of nodes and edges in CensNet is inspired by line graph and driven by mutually-enriched feature propagation rules, which can handle discrete or continuous node/edge features. 


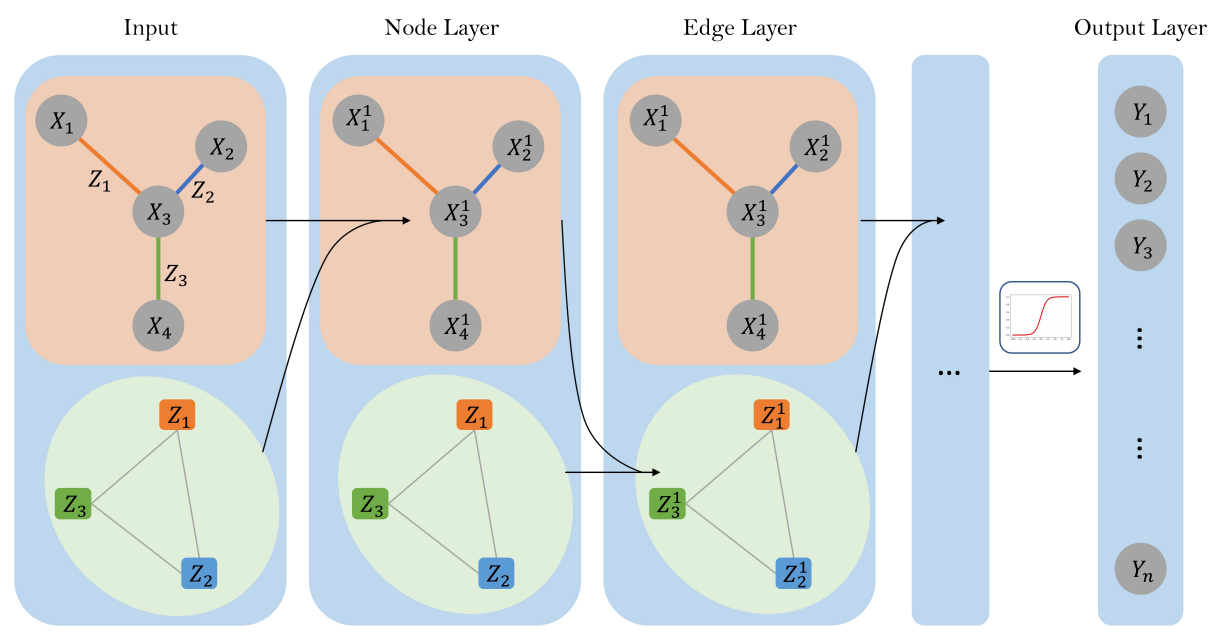

Figure 1: Illustrative depiction of Convolution with Edge-Node Switching (CensNet). The upper (orange color) components are convolution operations on node adjacency matrix and node features, while the lower (green color) components are the corresponding line graph convolution. Two types of layers as a combo, (1). Node Layer, update the node embedding with node and edge embedding from the previous layer, and (2). Edge Layer, update the edge embedding with the edge and node embedded features from the preceding layer.

\section{Convolution with Edge-Node Switching}

The CensNet framework consists of two types of layers, node layer and edge layer. Figure 1 shows the CensNet architecture for semi-supervised node classification. The input layer comprises of a node adjacency matrix and the corresponding node features, as well as its line graph counterpart - the edge adjacency matrix and edge features. The three colored edges (i.e., $z_{1}, z_{2}$ and $z_{3}$ ) in the sample graph are converted to three line graph nodes (squared shape). We define a CensNet combo as two types of layers, node layer and edge layer. In the node layer, all input data are processed to update the node embedding, while keeping the line graph (edge adjacency matrix and edge features) flow forward without any change. In the edge layer, we combine the updated node embedding with the line graph to update the edge embedding. Depending on the specific task and the availability of labels, CensNet adopts different types of activation functions for the node or edge embedding matrices. For example, in the graph node semi-classification task (e.g., paper classification in citation networks), we have the label for each node, and thus we can use the sigmoid function for the node embedding matrix in the final layer. For the graph classification or regression task (e.g., the molecular property prediction), we may apply an average pooling layer to reshape the node embeddings and obtain graph-level embeddings.

We should note, the CensNet framework is a high-level abstract of the interactive graph embedding with both nodes and edges. One does not necessarily use the approximated spectral graph kernel for the convolution. Other new techniques such as the kernels in Lanczos Network [Liao et al., 2019] can also be applied to our framework.

\subsection{Propagation Rules}

The CensNet uses approximated spectral graph convolution in the layer-wise propagation. We define the normalized
(Laplacianized) node adjacency matrix with self-loop as

$$
\tilde{A}_{v}=D_{v}^{-\frac{1}{2}}\left(A_{v}+I_{N_{v}}\right) D_{v}^{-\frac{1}{2}}
$$

where $D_{v}$ is the diagonal degree matrix of $A_{v}+I_{N_{v}}$, and $I$ is an identity matrix.

Propagation rule for node layer. The layer-wise propagation rule for node feature in the $(l+1)$-th layer is defined as

$$
H_{v}^{(l+1)}=\sigma\left(T \Phi\left(H_{e}^{(l)} P_{e}\right) T^{T} \odot \tilde{A}_{v} H_{v}^{(l)} W_{v}\right)
$$

where the matrix $T \in \mathbb{R}^{N_{v} \times N_{e}}$ is a binary transformation matrix and $T_{i, m}$ represents whether edge $m$ connects node $i$. Given the fact that each edge is formed by two nodes, each column of matrix $T$ has two elements being 1 and all others are $0 . P_{e}$ is a $d_{e}$-dimensional vector, defined as the learnable weights for edge feature vectors. $\Phi$ denotes the diagonalization operation, which places a onedimensional vector to the diagonals of a square matrix. $\odot$ denotes the Hadamard product or element-wise product. Another view of this rule is to map corresponding elements from $T \Phi\left(H_{e}^{(l)} P_{e}\right) T^{T}$ to the normalized node adjacency matrix, and the $T \Phi\left(H_{e}^{(l)} P_{e}\right) T^{T} \odot \tilde{A}_{v}$ is a fused node adjacency matrix by using information from the line graph counterpart. The line graph brings zero impact if there is no physical edge, and thus we maintain the sparsity of the original graph, bringing significant computing benefits.

Propagation rule for edge layer. Similarly, the normalized (Laplacianized) edge adjacency matrix is defined as

$$
\tilde{A}_{e}=D_{e}^{-\frac{1}{2}}\left(A_{e}+I_{N_{e}}\right) D_{e}^{-\frac{1}{2}},
$$

where $D_{e}$ is the degree matrix of $A_{v}+I_{N_{v}}$. Furthermore, we define the propagation rule for edge features as follows

$$
H_{e}^{(l+1)}=\sigma\left(T^{T} \Phi\left(H_{v}^{(l)} P_{v}\right) T \odot A_{e} H_{e}^{(l)} W_{e}\right) .
$$


The $T$ matrix is the same as Equation (2), while $P_{v}$ represents the learnable weight for the nodes. As a return, the node feature and adjacency matrix are used to improve the edge embedding. These two components bridge signals on nodes and edges, and the node and edge embeddings are updated alternatively.

\subsection{Task-Dependent Loss Functions}

The designs of the output layer, as well as the loss function, are task dependent. For node or edge classification tasks, we may apply the sigmoid function to the final hidden node or edge layers; for the graph classification task, we need an extra pooling operation that maps the node-level embeddings to a graph-level representation. For example, we take the average prediction of the atoms in a molecule as the graph-level output. The graph-level max pooling might not be appropriate from a practical point of view; a learnable parameter (weight) for each node is also not acceptable because of the tendency to overfitting.

For the semi-supervised node classification task, the loss function is defined as

$$
\mathcal{L}(\Theta)=-\sum_{l \in Y_{L}} \sum_{f=1}^{F} Y_{l f} \log M_{l f}
$$

where $Y_{L}$ is a set of nodes with labels, and $M$ is the softmax results of the output from the last node layer, assuming the final node feature map has $F$ dimensions. The $\Theta=$ $\left(W_{v}, W_{e}, P_{v}, P_{e}\right)$ is the parameter set. For multi-task graph classification, we may sum up the cross entropy from each target.

For regression task where the response is a continuous variable, we define the following loss function by using the $l^{p}$ regularized mean square error (MSE)

$$
\mathcal{L}(\Theta)=\sum_{l \in Y_{L}} \sum_{f=1}^{F}\left\|Y_{l f}-\hat{Y}_{l f}\right\|_{2}^{2}+\lambda\|\Theta\|_{p}
$$

where $\hat{Y}$ is the predicted outcome from the last node hidden layer. The regularized term is used to control the model complexity and avoid overfitting.

\subsection{Training Algorithms}

We show our algorithm for semi-supervised node classification in Algorithm 1, which uses the layer-wise propagation rules defined in Equations (2) and (4). Optimization algorithms, such as Adam or SGD, are employed to deal with the cross-entropy loss function. When the input data cannot fit into the GPU memory, a mini-batch strategy is usually preferred [Kingma and $\mathrm{Ba}, 2014]$. Thus, we also design a mini-batch training algorithm for large graphs in Algorithm 2 for the node classification task with few labeled nodes. The key idea is to sample the nodes from training, validation, and test set proportionally, to construct the batches. Our empirical results show that such a training strategy could match the performance of training with the entire graph.

\section{Experiments}

We evaluate the proposed CensNet method for three tasks on five benchmark data sets.

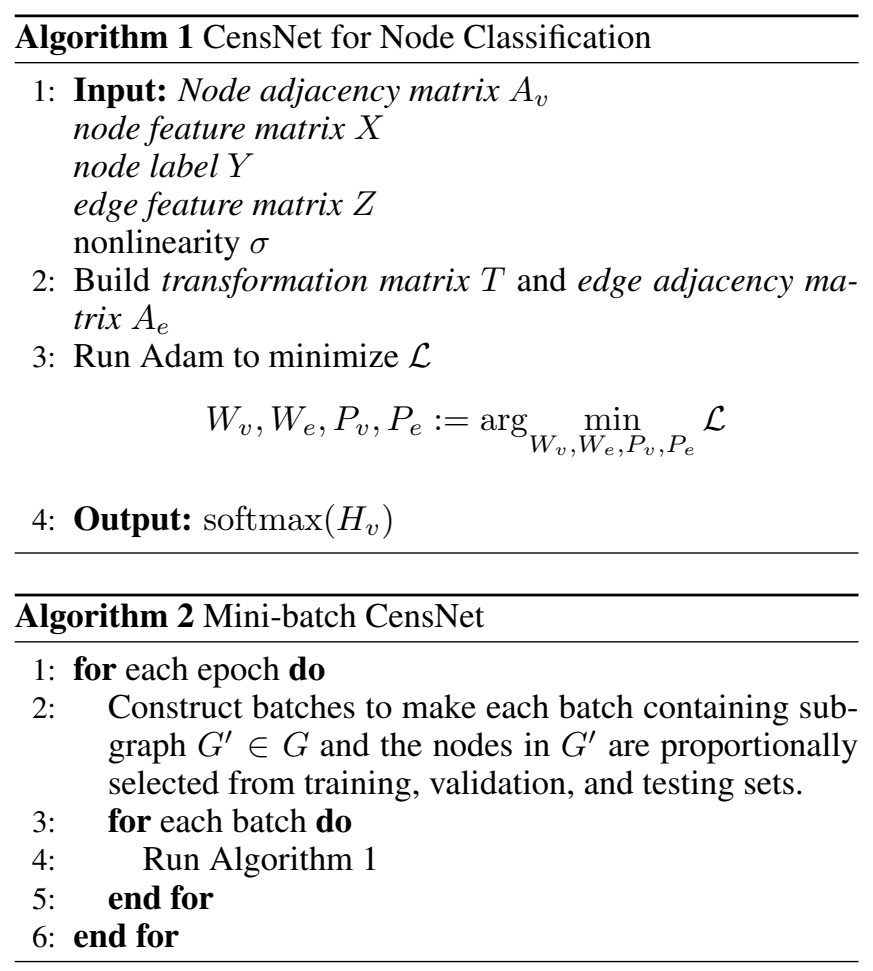

\subsection{Data Description and Preprocessing}

Tox21. The Toxicology in the 21st Century (Tox21, [Wu et al., 2018]) initiative created a public database measuring toxicity of compounds, which has been used in the 2014 Tox21 Data Challenge. This dataset contains quantitative toxicity measurements for 7,831 environmental compounds and drugs. A compound structure (in SMILE format) is usually expressed as a graph with atoms as nodes and bonds being edges. There are 55 bond features, and each atom has 25 features. Each compound is associated with 12 binary labels that represent the outcome (active/inactive) of 12 different toxicological experiments. There are about $20 \%$ missing values in these labels, and we exclude those observations when computing the loss but still keep them in the training process.

Lipophilicity. The Lipophilicity is an important feature of drug molecules that affects both membrane permeability and solubility [Wu et al., 2018]. This dataset provides experimental results of octanol/water distribution coefficient $(\log \mathrm{D}$ at $\mathrm{pH} 7.4$ ) of 4,200 compounds. There are 34 features on the edge (bond) and 25 features on the node (atom), while the response is one single continuous variable.

We follow the preprocessing steps in [Wu et al., 2018] to convert each compound in Tox 21 and Lipophilicity to a small graph, and remove the compounds whose SMILE representation cannot be converted to a graph structure. We then randomly split the dataset to different partitions as training, validation and test sets, respectively. We consider 4 data splitting settings, by keeping $60 \%, 70 \%, 80 \%$, and $90 \%$ of the molecule graphs as the training set, while equally breaking the rest of the data sets as validation and test sets.

Cora, Citeseer, and PubMed. These three data sets are benchmarks for the semi-supervised node classification task, 
Proceedings of the Twenty-Eighth International Joint Conference on Artificial Intelligence (IJCAI-19)

\begin{tabular}{|c|c|c|c|c|c|c|c|c|c|}
\hline Train & Data & \multicolumn{4}{|c|}{ Tox21 (AUC) } & \multicolumn{4}{|c|}{ Lipophilicity (RMSE) } \\
\cline { 3 - 9 } PCT & Split & RF & Logistic & GCN & CensNet & RF & LR & GCN & CensNet \\
\hline \multirow{2}{*}{$60 \%$} & Val. & $0.69 \pm 0.01$ & $0.69 \pm 0.01$ & $0.72 \pm 0.00$ & $\mathbf{0 . 7 6} \pm \mathbf{0 . 0 0}$ & $1.19 \pm 0.01$ & $1.46 \pm 0.37$ & $1.04 \pm 0.01$ & $\mathbf{0 . 9 4} \pm \mathbf{0 . 0 1}$ \\
& Test & $0.71 \pm 0.01$ & $0.71 \pm 0.01$ & $0.73 \pm 0.00$ & $\mathbf{0 . 7 7} \pm \mathbf{0 . 0 0}$ & $1.16 \pm 0.02$ & $1.17 \pm 0.03$ & $1.06 \pm 0.00$ & $\mathbf{0 . 9 7} \pm \mathbf{0 . 0 1}$ \\
\hline \hline \multirow{2}{*}{$70 \%$} & Val. & $0.70 \pm 0.01$ & $0.70 \pm 0.01$ & $0.73 \pm 0.00$ & $\mathbf{0 . 7 6} \pm \mathbf{0 . 0 0}$ & $1.18 \pm 0.02$ & $1.19 \pm 0.01$ & $1.02 \pm 0.01$ & $\mathbf{0 . 9 2} \pm \mathbf{0 . 0 1}$ \\
& Test & $0.70 \pm 0.01$ & $0.71 \pm 0.01$ & $0.74 \pm 0.00$ & $\mathbf{0 . 7 7} \pm \mathbf{0 . 0 0}$ & $1.16 \pm 0.02$ & $1.17 \pm 0.04$ & $1.05 \pm 0.01$ & $\mathbf{0 . 9 5} \pm \mathbf{0 . 0 1}$ \\
\hline \hline \multirow{2}{*}{$80 \%$} & Val. & $0.71 \pm 0.01$ & $0.71 \pm 0.01$ & $0.72 \pm 0.00$ & $\mathbf{0 . 7 6} \pm \mathbf{0 . 0 0}$ & $1.17 \pm 0.02$ & $1.16 \pm 0.02$ & $1.05 \pm 0.01$ & $\mathbf{0 . 9 6} \pm \mathbf{0 . 0 1}$ \\
& Test & $0.71 \pm 0.01$ & $0.71 \pm 0.01$ & $0.75 \pm 0.00$ & $\mathbf{0 . 7 8} \pm \mathbf{0 . 0 0}$ & $1.16 \pm 0.01$ & $1.15 \pm 0.01$ & $1.05 \pm 0.01$ & $\mathbf{0 . 9 3} \pm \mathbf{0 . 0 1}$ \\
\hline \hline \multirow{2}{*}{$90 \%$} & Val. & $0.71 \pm 0.02$ & $0.71 \pm 0.01$ & $0.74 \pm 0.00$ & $\mathbf{0 . 7 8} \pm \mathbf{0 . 0 1}$ & $1.18 \pm 0.02$ & $1.18 \pm 0.03$ & $1.08 \pm 0.00$ & $\mathbf{0 . 9 4} \pm \mathbf{0 . 0 2}$ \\
& Test & $0.71 \pm 0.02$ & $0.71 \pm 0.02$ & $0.75 \pm 0.00$ & $\mathbf{0 . 7 9} \pm \mathbf{0 . 0 1}$ & $1.13 \pm 0.03$ & $1.13 \pm 0.03$ & $0.97 \pm 0.00$ & $\mathbf{0 . 8 3} \pm \mathbf{0 . 0 2}$ \\
\hline
\end{tabular}

Table 1: Experimental results on Tox21 and Lipophilicity data sets.

which has been analyzed by many graph convolutional network models such as the ones in [Defferrard et al., 2016a; Kipf and Welling, 2017; Hamilton et al., 2017; Veličković et al., 2018; Liao et al., 2019]. Cora has 2,708 nodes (papers) and 5,429 edges (citation links), and each node has 1,433 tfidf features. The papers are classified to 7 different research areas thus the response has seven different values. Citeseer has 3,327 nodes and 4,732 edges with 3,703 node features; the papers are grouped into 6 research fields. PubMed contains 19,717 nodes and 44,338 edges, each node has 500 features and the papers are in 3 categories. Please remark that these citation graphs are not naturally good benchmarks for CensNet because there is no available edge feature. However, we still run our algorithm with effortless hand-crafted edge features to show the competitive performance of CensNet. Examples of such edge features could be the pairwise node feature correlations or cosine similarities.

For three citation graphs, we create two simple edge features: (1) the pairwise cosine similarities between corresponding node features, and (2) a 2-dimensional vector to represent the edge directions. If paper A cites paper B then the vector is $[1,0]$, otherwise $[0,1]$. We follow the splitting strategy in [Liao et al., 2019] and implement the experiments for different label rate. We evaluate our method and baselines with 3\%, $1 \%$ and $0.5 \%$ labeled data in training set on Cora, $1 \%, 0.5 \%$ and $0.3 \%$ on Citeseer and $0.1 \%, 0.05 \%$ and $0.03 \%$ on PubMed. For all three data sets, we randomly select 50\% for validation and the rest for testing.

\subsection{Experimental Settings}

Three tasks are implemented in our experiments.

Multi-task graph classification. We evaluate the performance of CensNet on the Tox 21 dataset under 4 data splitting scenarios. [Wu et al., 2018] presented a comprehensive model comparisons for the Tox21 data, and the state-ofthe-art method is GCN [Kipf and Welling, 2017]. We implemented the minibatch GCN, and other two classical classification algorithms, logistic regression and random forest, as three baselines. We report the area under the ROC curve (AUC) metric in both validation and test data sets for all compared methods.

Graph regression. The regression task is similar to graph classification but using different loss function and performance metric. We use the same data splitting strategies as in Tox21. The baseline algorithms are GCN, linear regres- sion, and random forest regression. We also report the root mean square error (RMSE) in both validation and test sets.

Semi-supervised node classification. This is a classical task in graph learning and statistical learning communities, and the most widely used benchmark data sets are three citation networks, Cora, Citeseer and PubMed. We adopt a few-shot learning strategy, i.e., only keep a small number of labeled data in training set while splitting the rest of the data for validation and test. We compare CensNet with seven representative graph convolution networks when using different percentages of labeled data. The seven baselines include ChebyNet [Defferrard et al., 2016b], GCN [Kipf and Welling, 2017], GraphSAGE [Hamilton et al., 2017], GAT [Veličković et al., 2018], LNet and AdaLet [Liao et al., 2019]. To the best of our knowledge, LNet and AdaLet are the state-of-the-art methods for this task [Liao et al., 2019], and we will present a comprehensive comparison between these two methods and the proposed CensNet.

All experiments are conducted on an Azure Linux VM (CPU: Intel(R) Xeon(R) CPU E5-2690 v3, GPU: NVIDIA Tesla K80). We implemented all graph convolution network algorithms in PyTorch [Paszke et al., 2017] v1.0.0. For other classical algorithms (random forest, linear regression, logistic regression), we used the implementations in the Python package Scikit-learn [Pedregosa et al., 2011]. For graph convolution models, we didn't implement any sophisticated fine-tuning strategies but tried different settings of learning rate from $\{0.01,0.005,0.001,0.0005\}$, batch size $\{16,32,64,128,256\}$, number of epochs $\{200,300,500,1000\}$, etc. We implemented three layers architecture (Node Layer - Edge Layer - Node Layer) with 32 units in each hidden layer. We report the best-performed results for each algorithm. For tree-based methods, we use cross-validation to tune the parameters; for linear and logistic regression models, we run the algorithms without using variable selection.

\subsection{Results and Discussions}

The experimental results show that the CensNet has competitive and consistent performance over diverse learning tasks.

Multi-task graph classification. Table 1 shows the AUC values on the validation and test sets, with four different training label rates. We replicate all experiments three times and report the mean and standard deviation of AUC values. The CensNet algorithm maintains significant advantages over all 
Proceedings of the Twenty-Eighth International Joint Conference on Artificial Intelligence (IJCAI-19)

\begin{tabular}{|c|c|c|c|c|c|c|c|c|}
\hline Data & TrainPCT & ChebyNet & GCN & GraphSAGE & GAT & LNet & AdaLNet & CensNet (Ours) \\
\hline \multirow{3}{*}{ Cora } & $3 \%$ & $62.1 \pm 6.7$ & $74.0 \pm 2.8$ & $64.2 \pm 4.0$ & $56.8 \pm 7.9$ & $76.3 \pm 2.3$ & $77.7 \pm 2.4$ & $\mathbf{7 9 . 4} \pm \mathbf{1 . 0}$ \\
& $1 \%$ & $44.2 \pm 5.6$ & $61.0 \pm 7.2$ & $49.0 \pm 5.8$ & $48.6 \pm 8.0$ & $66.1 \pm 8.2$ & $\mathbf{6 7 . 5} \pm \mathbf{8 . 7}$ & $67.1 \pm 1.3$ \\
& $0.5 \%$ & $33.9 \pm 5.0$ & $52.9 \pm 7.4$ & $37.5 \pm 5.4$ & $41.4 \pm 6.9$ & $58.1 \pm 8.2$ & $\mathbf{6 0 . 8} \pm \mathbf{9 . 0}$ & $57.7 \pm 3.9$ \\
\hline \hline \multirow{3}{*}{ Citeseer } & $1 \%$ & $59.4 \pm 5.4$ & $58.3 \pm 4.0$ & $51.0 \pm 5.7$ & $46.5 \pm 9.3$ & $61.3 \pm 3.9$ & $\mathbf{6 3 . 3} \pm \mathbf{1 . 8}$ & $62.5 \pm 1.5$ \\
& $0.5 \%$ & $45.3 \pm 6.6$ & $47.7 \pm 4.4$ & $33.8 \pm 7.0$ & $38.2 \pm 7.1$ & $53.2 \pm 4.0$ & $53.8 \pm 4.7$ & $\mathbf{5 7 . 6} \pm \mathbf{3 . 0}$ \\
& $0.3 \%$ & $39.3 \pm 4.9$ & $39.2 \pm 6.3$ & $25.7 \pm 6.1$ & $30.9 \pm 6.9$ & $44.4 \pm 4.5$ & $46.7 \pm 5.6$ & $\mathbf{4 9 . 4} \pm \mathbf{3 . 6}$ \\
\hline \hline \multirow{3}{*}{ PubMed } & $0.1 \%$ & $55.2 \pm 6.8$ & $73.0 \pm 5.5$ & $65.4 \pm 6.2$ & $59.6 \pm 9.5$ & $\mathbf{7 3 . 4} \pm \mathbf{5 . 1}$ & $72.8 \pm 4.6$ & $69.9 \pm 2.1$ \\
& $0.05 \%$ & $48.2 \pm 7.4$ & $64.6 \pm 7.5$ & $53.0 \pm 8.0$ & $50.4 \pm 9.7$ & $\mathbf{6 8 . 8} \pm \mathbf{5 . 6}$ & $66.0 \pm 4.5$ & $65.7 \pm 1.2$ \\
& $0.03 \%$ & $45.3 \pm 4.5$ & $57.9 \pm 8.1$ & $45.4 \pm 5.5$ & $50.9 \pm 8.8$ & $60.4 \pm 8.6$ & $61.0 \pm 8.7$ & $\mathbf{6 1 . 4} \pm \mathbf{2 . 8}$ \\
\hline
\end{tabular}

Table 2: Node classification accuracy (in percent) on three citation graph data sets.

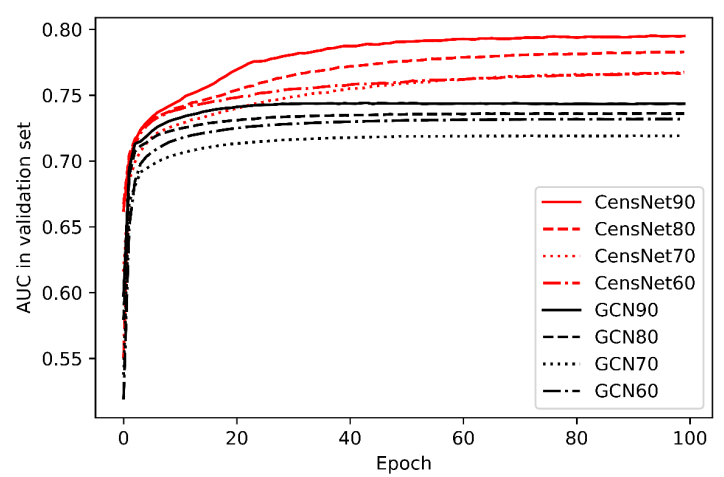

(a)

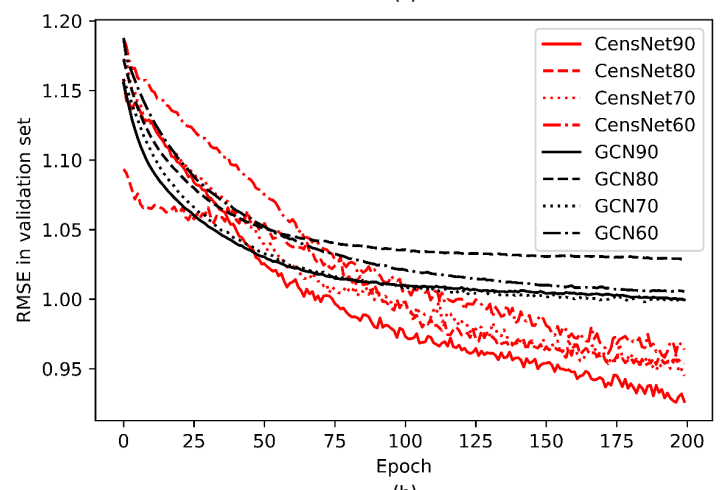

(b)

Figure 2: AUC/RMSE in validation set for Tox21/Lipophilicity. The name of each curve is formed with algorithm name and label ratio in training set. For example, CensNet90 means the CensNet algorithm with $90 \%$ data in training set.

other methods in all settings, while both GCN and CensNet perform better than the other two traditional methods. The logistic regression and random forest models can hardly capture the association between signals and response, even with increased training sets. Figure 2(a) shows the changes in validation AUC in 200 epochs. The GCN's curve becomes flat within 20 epochs while the CensNet can continuously improve. Also, the CensNet with smaller training sets can beat the GCN with more training data, uniformly and consistently.

Graph regression. Compared with three baseline methods, our CensNet has achieved the best performance in both validation and test sets under four training settings. For a fair comparison, we replicate all experiments three times and report the mean RMSE with standard errors. The following conclusions can be summarized from Table 1: (1). all these
4 algorithms achieve better performance with larger training sets, while random forest and logistic regression only obtain limited performance lift. (2). both GCN and CensNet have large positive margins over traditional methods, indicating that the graph structure is not neglectable in the molecule regression task. (3). CensNet improves the performance of GCN by $5 \%-15 \%$ in RMSE, which implies that considering the edge features in the molecule can improve the quality of node embedding, as a consequence, bring significant benefits to the learning process. Figure 2(b) shows that the CensNet gains leading positions after around 50-75 epochs, while GCN's curves still keep flat.

Semi-supervised node classification. We follow the same experimental settings in [Liao et al., 2019] and re-use the benchmark results as our baselines. For all the experiments, we observed significant overfitting with low label rates for all graph convolution networks. We highlight the bestperformed method (with the highest accuracy in the test set) in each setting for all three data sets. Our CensNet method obtains the best accuracy in 4 out of 9 experiments, which is followed by LNet and AdaLNet - the state-of-the-art algorithms for this task. One may believe that adding edge information to the algorithm is not a fair comparison to the benchmarks; however, our newly created edge features are all from the benchmark data; thus no extra signal is introduced. The classical GCN [Kipf and Welling, 2017] also achieves competitive results in most scenarios, which coincides the conclusion from the extensive experiments in [Shchur et al., 2018].

\section{Conclusion}

Graph convolution models open up an exciting area of deep learning in non-Euclidean space. Our newly proposed CensNet framework established a novel approach to learn the node and edge feature embeddings simultaneously. The extensive experiments on five benchmark data sets show that the proposed CensNet algorithm can achieve the state-of-art performance in three major graph learning tasks. We are currently exploring other graph kernels and more efficient training algorithms to scale up the deep graph learning to larger and more complex data sets.

\section{Acknowledgments}

The authors thank the anonymous reviewers for their helpful comments. This work was supported in part by a gift from Adobe. 


\section{References}

[Bronstein et al., 2017] Michael M Bronstein, Joan Bruna, Yann LeCun, Arthur Szlam, and Pierre Vandergheynst. Geometric deep learning: going beyond euclidean data. IEEE Signal Processing Magazine, 34(4):18-42, 2017.

[Defferrard et al., 2016a] Michaël Defferrard, Xavier Bresson, and Pierre Vandergheynst. Convolutional neural networks on graphs with fast localized spectral filtering. In D. D. Lee, M. Sugiyama, U. V. Luxburg, I. Guyon, and R. Garnett, editors, Advances in Neural Information Processing Systems 29, pages 3844-3852. Curran Associates, Inc., 2016.

[Defferrard et al., 2016b] Michaël Defferrard, Xavier Bresson, and Pierre Vandergheynst. Convolutional neural networks on graphs with fast localized spectral filtering. In NIPS, 2016.

[Hamilton et al., 2017] William L. Hamilton, Rex Ying, and Jure Leskovec. Inductive representation learning on large graphs. In NIPS, 2017.

[Harary and Norman, 1960] Frank Harary and Robert Z. Norman. Some properties of line digraphs. Rendiconti del Circolo Matematico di Palermo, 1960.

[He et al., 2016] Kaiming He, Xiangyu Zhang, Shaoqing Ren, and Jian Sun. Deep residual learning for image recognition. 2016 IEEE Conference on Computer Vision and Pattern Recognition (CVPR), pages 770-778, 2016.

[Ji and Jin, 2016] Pengsheng Ji and Jiashun Jin. Coauthorship and citation networks for statisticians. The Annals of Applied Statistics, 10(4):1779-1812, 2016.

[Kingma and $\mathrm{Ba}, 2014]$ Diederik P. Kingma and Jimmy Ba. Adam: A method for stochastic optimization. CoRR, abs/1412.6980, 2014.

[Kipf and Welling, 2017] Thomas N. Kipf and Max Welling. Semi-supervised classification with graph convolutional networks. In International Conference on Learning Representations, 2017.

[Krizhevsky et al., 2012] Alex Krizhevsky, Ilya Sutskever, and Geoffrey E. Hinton. Imagenet classification with deep convolutional neural networks. In Proceedings of the 25th International Conference on Neural Information Processing Systems - Volume 1, NIPS'12, pages 1097-1105, USA, 2012. Curran Associates Inc.

[LeCun et al., 2015] Yann LeCun, Yoshua Bengio, and Geoffrey Hinton. Deep learning. Nature, 521(7553):436444, 52015.

[Li and Fu, 2015] Sheng Li and Yun Fu. Learning balanced and unbalanced graphs via low-rank coding. IEEE Transactions on Knowledge and Data Engineering, 27(5):12741287, 2015.

[Li et al., 2017] Sheng Li, Hongfu Liu, Zhiqiang Tao, and Yun Fu. Multi-view graph learning with adaptive label propagation. In IEEE International Conference on Big Data, pages 110-115. IEEE, 2017.
[Liao et al., 2019] Renjie Liao, Zhizhen Zhao, Raquel Urtasun, and Richard Zemel. Lanczosnet: Multi-scale deep graph convolutional networks. In International Conference on Learning Representations, 2019.

[Monti et al., 2017] Federico Monti, Davide Boscaini, Jonathan Masci, Emanuele Rodola, Jan Svoboda, and Michael M. Bronstein. Geometric deep learning on graphs and manifolds using mixture model cnns. In The IEEE Conference on Computer Vision and Pattern Recognition (CVPR), July 2017.

[Namata et al., 2012] Galileo Mark Namata, Ben London, Lise Getoor, and Bert Huang. Query-driven active surveying for collective classification. In Workshop on Mining and Learning with Graphs (MLG), 2012.

[Paszke et al., 2017] Adam Paszke, Sam Gross, Soumith Chintala, Gregory Chanan, Edward Yang, Zachary DeVito, Zeming Lin, Alban Desmaison, Luca Antiga, and Adam Lerer. Automatic differentiation in pytorch. NIPS$W, 2017$.

[Pedregosa et al., 2011] F. Pedregosa, G. Varoquaux, A. Gramfort, V. Michel, B. Thirion, O. Grisel, M. Blondel, P. Prettenhofer, R. Weiss, V. Dubourg, J. Vanderplas, A. Passos, D. Cournapeau, M. Brucher, M. Perrot, and E. Duchesnay. Scikit-learn: Machine learning in Python. Journal of Machine Learning Research, 12:2825-2830, 2011.

[Scarselli et al., 2009] F. Scarselli, M. Gori, A. C. Tsoi, M. Hagenbuchner, and G. Monfardini. The graph neural network model. IEEE Transactions on Neural Networks, 20(1):61-80, Jan 2009.

[Schlichtkrull et al., 2018] Michael Sejr Schlichtkrull, Thomas N. Kipf, Peter Bloem, Rianne van den Berg, Ivan Titov, and Max Welling. Modeling relational data with graph convolutional networks. In ESWC, 2018.

[Sen et al., 2008] Prithviraj Sen, Galileo Mark Namata, Mustafa Bilgic, Lise Getoor, Brian Gallagher, and Tina Eliassi-Rad. Collective classification in network data. AI Magazine, 29(3):93-106, 2008.

[Shchur et al., 2018] Oleksandr Shchur, Maximilian Mumme, Aleksandar Bojchevski, and Stephan Günnemann. Pitfalls of graph neural network evaluation. CoRR, abs/1811.05868, 2018.

[Veličković et al., 2018] Petar Veličković, Guillem Cucurull, Arantxa Casanova, Adriana Romero, Pietro Liò, and Yoshua Bengio. Graph attention networks. In International Conference on Learning Representations, 2018.

[Wu et al., 2018] Zhenqin Wu, Bharath Ramsundar, Evan N. Feinberg, Joseph Gomes, Caleb Geniesse, Aneesh S. Pappu, Karl Leswing, and Vijay Pande. Moleculenet: a benchmark for molecular machine learning. Chem. Sci., 9:513-530, 2018.

[Zhou et al., 2018] Jie Zhou, Ganqu Cui, Zhengyan Zhang, Cheng Yang, Zhiyuan Liu, and Maosong Sun. Graph neural networks: A review of methods and applications. CoRR, abs/1812.08434, 2018. 